\title{
Theory of Unidirectional and Multidirectional Forces and Violation of Third Law of Motion
}

\author{
Debjyoti Biswadev Sengupta \\ Student-Class XI, Smt. Sulochanadevi Singhania School \\ Home: Flat 404, Building 28, Vijay Annex, Waghbil, Ghodbunder Road, Thane(W), Maharashtra, India - 400615 \\ School: Smt. Sulochanadevi Singhania School, Jekegram, Thane(W), Maharashtra, India - 400606
}

\begin{abstract}
This research paper introduces the idea of force on the basis of their direction/nature of the forces acting on the bodies. This paper will also make an attempt to answer the unanswered with regards to gravitational force.
\end{abstract}

Keywords: forces, unidirectional, multidirectional, gravitation, electrostatics, magnetism, non-contact forces

\section{Introduction}

It is thought that there are various contact forces and three non-contact forces which exist in the universe, namely the electrostatic force, the magnetic force, and the force of gravitation. As per this theory, it is considered that the force of gravitation is the most mysterious of all the non-contact forces. There are few things which neither the Newtonian concept of physics, nor the modern concept of physics has been able to explain, as far the force of gravitation is concerned. The author hypothesises that these unanswered queries may be answered if we explain the non-contact forces in terms of their conventional direction/nature of the forces. Hence, the idea of unidirectional and multidirectional forces is perceived.

The paper shall be dealt under 4 headings. Heading II will introduce the concept of a unidirectional and its characteristics. Heading III will deal with the introduction and characteristics of a multidirectional forces. Heading IV shall deal with the categorisation of the various forces which exists under Table I and Heading V shall deal with the explanation of the concept of multidirectional forces reasons of considering the force of gravitation as a multidirectional force. Heading VI shall deal with mathematical proof of noncontact forces violating Newton's Third Law of Motion. Heading VII will see the founding of new set of laws which are obeyed by multidirectional forces only. Heading VIII will deal with the applications and conclusion of the theory.

\section{Unidirectional Forces and Their Characteristics}

Sub-heading $\mathrm{A}$ is concerned with the introduction of the concept of unidirectional forces while sub-heading B is mainly concerned with the characteristics of unidirectional forces and its examples and exceptions (if any).
A. Introduction of Unidirectional Forces
Unidirectional forces can be defined as those forces which are either completely attractive in nature or completely repulsive in nature or is acting only towards a single direction. These forces have got their name from their characteristic of acting only in one direction.

B. Characteristics of Unidirectional Forces

- Unidirectional forces are ideal forces, better known as pseudoforces.

- Such forces do not exist in the universe. They exist only in the Earth.

- These forces are unperceivable by the human body.

- They are either completely attractive in nature or completely repulsive in nature or act only in one direction.

- Unidirectional forces are in accordance with Newton's Third Law of Motion which is stated in his book 'Principia Mathematica' as

"Lex III: Actioni contrariam semper et aqualem esse reactionem: sive corporum duorum actiones in se mutuo semper esse aquales et in partes contrarias dirigi." (in Latin)

Translated to English, this reads:

"Law III: To every action there is always opposed an equal reaction: or the mutual actions of two bodies upon each other are always equal, and directed to contrary parts."

Usually, all pseudo forces fall under the category of unidirectional forces. The force which is the most apt example of a unidirectional force is centrifugal force which is a pseudoforce, acts only in one direction, is unperceivable by the human body and obeys Newton's Third Law of Motion.

Unidirectional forces do have exceptions. The most apt example of a force which can be considered as a unidirectional force is the force of friction, which is a real force but acts only in the direction opposite to the applied force to resist the motion of the body. Thus, contact forces can be considered as unidirectional forces.

\section{Multidirectional Forces and Their Characteristics}

Sub-heading A is concerned with the introduction of the concept of multidirectional forces while sub-heading B is mainly concerned with the characteristics of multidirectional forces and its examples. 


\section{International Journal of Science and Research (IJSR) \\ ISSN (Online): 2319-7064}

Index Copernicus Value (2016): 79.57 | Impact Factor (2015): 6.391

\section{A. Introduction of Multidirectional Forces}

Multidirectional forces can be defined as those forces which are attractive as well as repulsive in nature or is acting towards two directions at the given instant of time. These forces have got their name from their characteristic of acting in both directions and their dual nature.

\section{B. Characteristics of Multidirectional Forces}

- Multidirectional forces are real forces.

- Such forces do exist in the universe.

- These forces are perceivable by the human body.

- They are attractive as well as repulsive in nature and is acting towards two directions.

- Multidirectional forces are in violation with Newton's Third Law of Motion which is stated in his book 'Principia Mathematica' as

"Lex III: Actioni contrariam semper et aqualem esse reactionem: sive corporum duorum actiones in se mutuo semper esse aquales et in partes contrarias dirigi." (in Latin)

Translated to English, this reads:

"Law III: To every action there is always opposed an equal reaction: or the mutual actions of two bodies upon each other are always equal, and directed to contrary parts."

It is found that multidirectional forces are not in accordance with Newton's Third Law. All non-contact forces are multidirectional in nature. The most apt example of a multidirectional force is the electrostatic force. It is found that these forces obey a different law.

\section{Categorization of Forces}

Table I

\begin{tabular}{|c|c|}
\hline Unidirectional Forces & Multidirectional Forces \\
\hline Frictional Force & Gravitational Force \\
\hline Tension Force & Electrostatic Force \\
\hline Normal Reaction Force & Magnetic Force \\
\hline Air Resistance & \\
\hline Applied Force & \\
\hline $\begin{array}{c}\text { Pseudoforce (Centrifugal } \\
\text { Force, Centripetal Force, etc.) }\end{array}$ & \\
\hline
\end{tabular}

This table categorises all the contact and non-contact forces under the categories of unidirectional and multidirectional forces.

\section{Concept of Multidirectional Force and Case Study of Gravitational Force}

Under this heading, we shall discuss the concept of multidirectional forces with few examples and also the reasons due to which gravitation force should be considered as a multidirectional force.
A. Concept of Multidirectional Force from examples Ex.1: Electrostatic forces between atoms

The most apt and simple example of a multidirectional force is the electrostatic force between two atoms.

In Figure I., there are two forces which are exerting electrostatic forces between each other and they are of the same element.

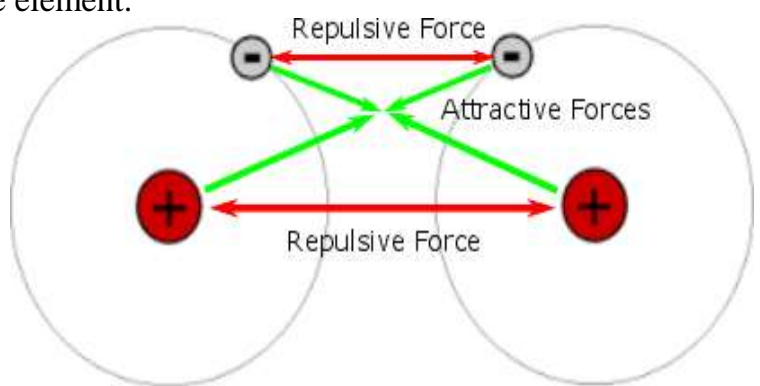

Figure 1: Electrostatic force between two atoms (Courtesy: Quora)

In the above figure, it is observed that the nucleus is exerting attractive forces on the electron of the other atom as well as the electron revolving around it. But the nucleus is exerting a repulsive force at the same instant on the nucleus of the other atom, and so do the electrons exert repulsive force on each other. Thus, electrostatic forces are multidirectional forces as the atoms are exerting attractive as well as repulsive forces on each other simultaneously at a given instant of time. In the above case, the net magnitudes of the attractive and repulsive forces are equal in magnitude, which keeps the nucleus and the electrons in their position. This example should not be confused with the formation of an ionic/covalent bond between two atoms. In this case, the net magnitude of the attractive forces are greater than the net magnitude of the repulsive forces.

\section{Ex.2: Magnetic Forces between two magnets.}

We can also explain a multidirectional force with the help of an example of a pair of magnets.

In the given figure II., we can see that the first pair of magnets is attracting each other whereas the second pair of magnets is repelling each other.

In the given figure, the first pair of magnets are attracting each other because the net magnitude of the attractive forces between the two poles is greater than the net magnitude of repulsive forces between the two poles. Since the net magnitude of repulsive forces between the two poles is considerably small as compared to the attractive forces, they may not be considered for the diagram. The second pair of magnets are repelling each other because the net magnitude of the repulsive forces between the two poles of the magnet is either equal or greater than the net magnitude of the attractive forces between the two poles of the magnet, depending on the strength of the two magnets taken. 


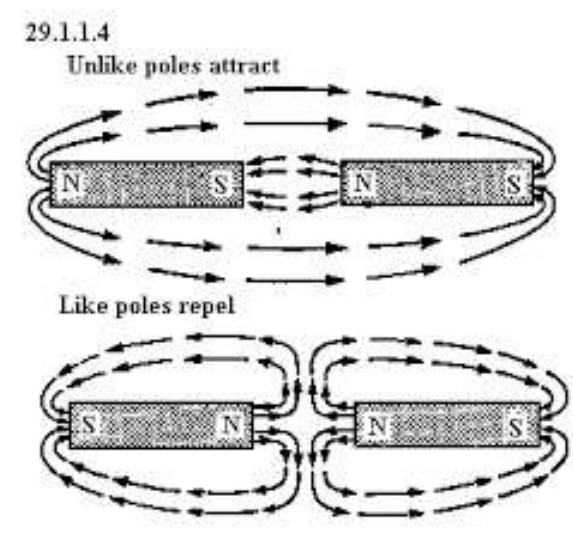

Figure II. Magnetic forces between two pair of magnets (Courtesy: University of Queensland, Australia)

B. Reasons to consider gravitational force as a multidirectional force.

The most intriguing question in this theory is whether gravitational force can be considered as a multidirectional force or a unidirectional force. Before we get into this debate, I will like to explain the example of a spring which is a unique structure as it can exhibit both unidirectional forces as well as multidirectional forces.

- Case I: When the one of the ends of the spring is fixed. When one of the ends of the spring is fixed to a rigid support and try to compress or stretch the spring within its limit of elasticity, we will observe that it will exert an equal and opposite restoring force which tells us that in this case, the spring is exerting a unidirectional force.

- Case II: When both ends of the spring is free to vibrate

When neither end of the spring is attached to the rigid support i.e. both ends are free to vibrate and the spring is compressed or stretched within its limit of elasticity, our naked eyes will deceive us in believing the fact that an equal and opposite force is acting on the spring, but actually there are two forces which are acting on the spring on either ends when the spring is trying to regain its original dimensions. This observation helps us in interpreting that a spring is not only capable of exhibiting unidirectional force but also multidirectional force which makes the spring a unique structure in terms of this theory.

Now, since we have completed the case study of the spring, we can get into the discussion of whether gravitational force is a unidirectional force or multidirectional force.

As per observations and this theory, gravitational force must be considered as a multidirectional force. The reason why the gravitational force should be considered a multidirectional force is as follows:
- Structure of the Gravitational wave

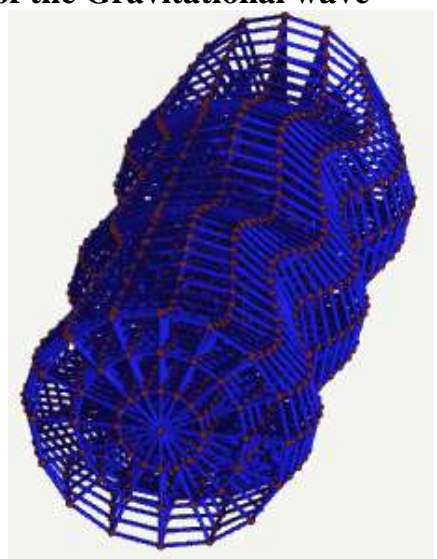

Figure III. Graphical Representation of the Structure of Gravitational Wave

(Courtesy: Max Planck Institute of Gravitational Physics)

As per the given graphical representation, we see that the gravitational waves resemble the structure of a spring as well as it behaves like a spring. The gravitational waves get compressed and stretched simultaneously at the same instant of time. The structure of the gravitational waves resembles more of the structure of a spring whose neither ends are attached to a rigid support. Hence, if we compare the structure of the gravitational waves to the structure of a free spring, we can easily interpret that the gravitational force is a type of multidirectional force as these waves exhibit multidirectional forces on two bodies.

\section{- Planetary orbits and planets.}

We observe that all planetary objects are following a constant imaginary elliptical path called as the orbits and none of the planetary objects leaves this imaginary path. Now, if the gravitational force would have been only attractive in nature, it would have caused all the planetary objects to get closer to each other causing a violent collision. Thus, we must understand that the planetary objects exhibit a force which is attractive as well as repulsive in nature which is none other than the gravitational force.

\section{- Magnetic field of planetary objects}

Quite similar to the discussion as in the second point, we know that every planetary object has its own magnetic field. Since it has its own magnetic field, we know that the planetary object will exhibit multidirectional force and the gravitational force is the multidirectional force specified here.

\section{Mathematical Proof of Multidirectional Forces Violating Newton's Third Law of Motion}

Here, we will prove that non-contact forces will violate Newton's Third Law of Motion and we will prove it separately for each non-contact force. 


\section{International Journal of Science and Research (IJSR) \\ ISSN (Online): 2319-7064}

Index Copernicus Value (2016): 79.57 | Impact Factor (2015): 6.391

- Proof I. Electrostatic force

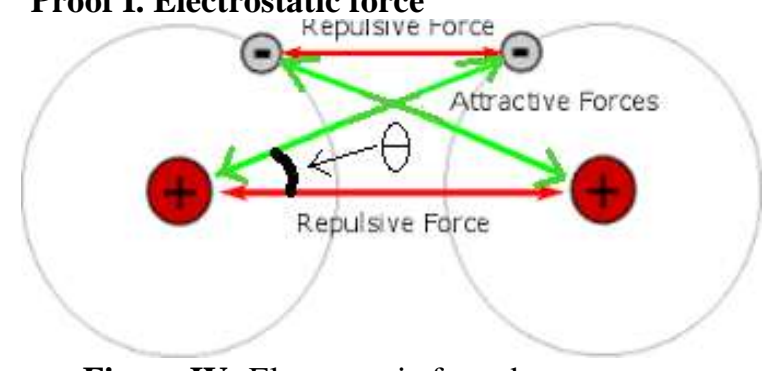

Figure IV: Electrostatic force between two atoms

As per the given diagram, we have four electrostatic forces acting, two each of electrostatic force of attraction as well as repulsion. In this case, we will take into consideration of hydrogen atom. So, let us proceed with the derivation.

Let $F_{1}$ and $F_{2}$ be the electrostatic force of repulsion

Let $F_{a}$ and $F_{4}$ be the electrostatic force of attraction

Let $r$ be the internuclear distance between the two atoms

Let $r^{\prime}$ be the distance between the nucleus and the electron

Let $q_{1}$ and $q_{2}$ be the charge of the proton and electron

$$
\begin{gathered}
\text { We know }, F=\frac{1}{4 \pi \varepsilon_{0}} \frac{q_{1} q_{2}}{r^{2}} \\
F_{1}=\frac{1}{4 \pi \varepsilon_{0}} \frac{q_{1} q_{2}}{r^{2}} \\
F_{1}=\frac{1}{4 \pi \varepsilon_{0}} \frac{\left(1 \cdot 6 \times 10^{-19}\right)^{2}}{r^{2}} \\
\text { Similarly. } F_{2}=\frac{1}{4 \pi \varepsilon_{0}} \frac{\left(-1 \cdot 6 \times 10^{-19}\right)^{2}}{r^{2}}
\end{gathered}
$$

Now, as per the given diagram, $r^{\prime}=\frac{r}{\cos \theta}$, where $\theta$ is angle between $r^{\prime}$ and $r$

$$
\begin{gathered}
F_{a}=\frac{1}{4 \pi \varepsilon_{0}} \frac{q_{1} q_{2}}{r^{2}} \\
F_{3}=\frac{1}{4 \pi \varepsilon_{0}} \frac{-\left(1 \cdot 6 \times 10^{-19}\right)^{2} \times \cos ^{2} \theta}{r^{2}}=F_{4}
\end{gathered}
$$

Let $F_{Y}$ be the net electrostatic force of repulsion Let $F_{\alpha}$ be the net electrostatic force of attraction

$$
\begin{gathered}
F_{r}=F_{1}+F_{2} \\
F_{a}=F_{a}+F_{4}
\end{gathered}
$$

As per the above calculations, the net electrostatic force of attraction between the atoms is more than the net electrostatic force of repulsion between the two atoms. As per Newton's Third Law of Motion,

$$
F_{Y}=F_{\alpha}
$$

but as per our calculations and observations,

$$
F_{Y} \neq F_{a}
$$

This show that electrostatic force, which is a non-contact force, disobeys Newton's Third Law of Motion.

\section{- Proof II. Gravitational Force}

Gravitational force is another non-contact force, also an important one, for which we will prove that it violates Newton's Third Law of Motion

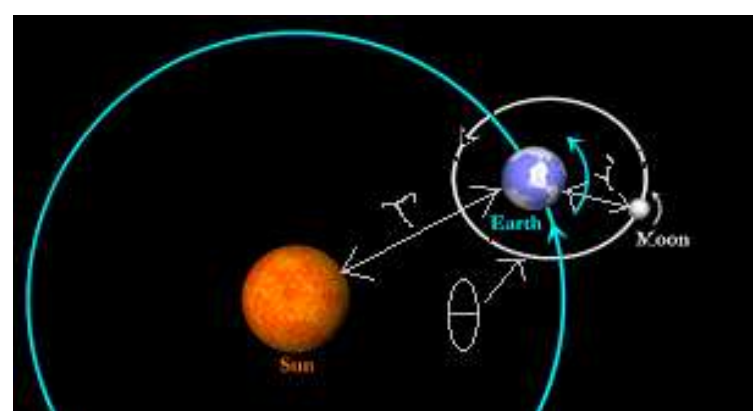

Figure V: Orbit diagram of the sun-earth-moon system

As per the given diagram, we will take into consideration of the net attractive force and repulsive force of the earth with respect to other planetary objects to make the derivation much simpler. Here, we are taking into consideration the sunearth-moon system for our derivation. Here, the earth is exerting an attractive force on the moon and a repulsive force on the sun. So, let us proceed with the derivation.

Let $F_{Y}$ be the net repulsive force of the earth

Let $F_{a}$ be the net atrractive force of the earth

Let $r$ be the distance between the sun and the earth

Let $r^{\prime \prime}$ be the distance between the earth and the moon

Let $\theta$ be the angle made by $r^{\prime \prime}$ with respect to $r$

Let $M_{e}$ be the mass of the earth

Let $M_{m}$ be the mass of the moon

Let $M_{g}$ be the mass of the Sun

$$
\begin{aligned}
& F=G \frac{m_{1} m_{2}}{r^{2}} \\
& F_{r}=G \frac{M_{g} M_{g}}{r^{2}} \\
& F_{a}=G \frac{M_{g} M_{m}}{(r)^{2}}
\end{aligned}
$$

Now, as per Newton's Third Law of Motion, the net attractive force of the earth should have been equal to the net repulsive force of the earth i.e.

$$
F_{T}=F_{0} \text {, }
$$

but as per our calculation and observation,

$$
F_{T} \neq F_{a} \text {. }
$$

This shows that the gravitational force also violates Newton's Third Law of Motion.

\section{Laws of Multidirectional Forces}

Under this heading, we shall see the foundation of the laws which are obeyed by all multidirectional forces (non-contact forces) only. The laws are as follows:

Law 1: All multidirectional forces have their own field and waves.

Law 2: All multidirectional forces are attractive and repulsive in nature.

Law 3: A body exerting a multidirectional force can exert forces of attractive as well as repulsive nature on two different bodies in different direction at the same instant of time.

Law 4: The net attractive force of the body may or may not be equal to the net repulsive force. 
Thus, in case of non-contact forces, "to every action, there may or may not be an equal and opposite reaction at the same instant of time".

\section{Applications and Conclusions}

As we come to the end of this theory, let us not the applications of the aforementioned theory.

- The theory will help us in understanding non-contact forces like gravitational forces from a different perspective.

- The theory can also help in producing results to experiments related to the discovery of dark matter and dark energy.

- The theory will defy the idea of gravitational force being a non-contact force of an attractive nature only.

- The theory may also help in explanation of certain phenomenon like the formation of tides, etc.

- The theory also lays foundation for a theory on the concept of chemical bonding.

Now, for conclusions, we can see and note them down as follows:

- Newton's Third Law of Motion is violated by all noncontact forces.

- All multidirectional forces obey a different set of laws.

- Newton's Third Law of Motion is not a universal law.

- Newton's Third Law of Motion is applicable only for contact forces.

\section{Author Profile}

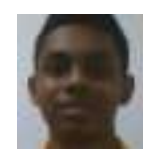

Debjyoti Biswadev Sengupta is a Grade 11 student in Smt. Sulochanadevi Singhania School, Thane(W), one of India's best ICSE schools. He published his first paper at the age of 17 titled 'Solving quadratic equations by calculus and its applications' in International Journal of Mathematics Trends and Technology. He currently lives in India. 